UC-21

LA $-9165-\mathrm{NS}$

Issued: January 1982

DE82 010845

\title{
Expansion of lons in Conducting Fluids With Imbedded Magnetic Fields
}

1. O. Bohachevsky

J. C. Goldstein

D. O. Dickman

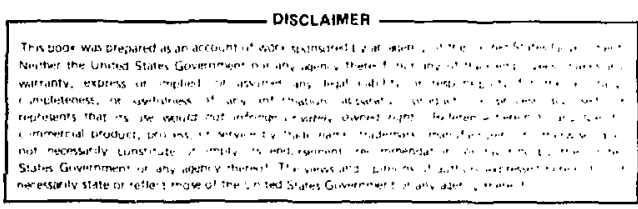

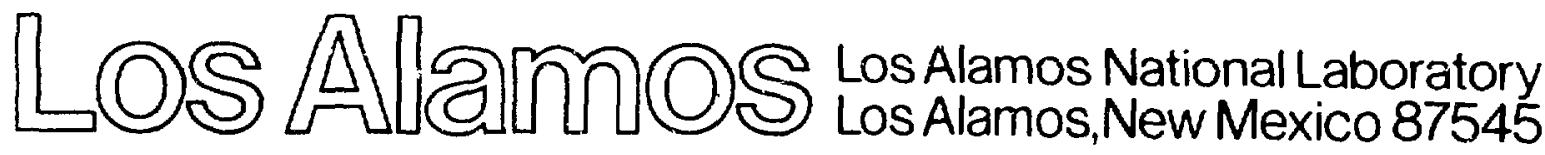


EXPANSION OF IONS IN CONDUCTING FLUIDS WITH IMBEDDED MAGNETIC FIELDS

by

I. O. Bohachersky, J. C. Goldstein, and D. O. Dickman

ABSTRACT

Studies of applications of inertial confinement fusion motivated the development of a plasma model composed of (1) a perfectly conducting fluid, (2) relatively energetic ions not in thermal squilibrium with the fluid, and (3) an electromagnetic field. Trajectories of the fons are determined from integration of the equations of motion for a statistically representative sample of simulation particles because the ion ranges in the fluid are comparable to characteristic dimensions of containment vessels. The model constituents interact through electrodynamic and collisional forces, and through jonization and/or recombination processes. The finite difference equations describing these interactions are integrated numerically for an isotropic ion source located on the cylinder axis. The solutions indicate distinctly different behaviors for low and high fluid densities. The ions expand in a diffuse cloud through low-density fluids, but in high-density fluids the ions aggregate into sheets that are perpendicular to the magnetic field.

\section{INTRODUCTION}

Applications of inertial confinement fusion (ICF), that is, fusion in which the reacting constituents are held together during the reaction by the force of inertia, have been studied at LoS Alamos for many years. In the ICF process the reaction occurs rapidly enough (in less than $10^{-9} \mathrm{~s}$ ) to resemble a small explosion (microexplosion), wich produces relatively high-energy (1-100 keV) ions. Investigations of the expansion of these ions and of their interaction with an externaliy generated magnetic field imbedded in a residual background fluid led to the construction and study of a plasma model composed of (1) an ionized flutd, ( $(\hat{)})$ relatively energetic ions not in thermodymamic equilibrium with the surrounding fluid, and (3) an electromagnetic field. Motivation, parameter estimates, and physical assumptions leading to this model have been published previously, $1-2$ together with the governing equations and the results that illustrate engineering implications for ICF applications.
In this paper we present (1) a summary description of the plasma model with the relevant parameter ranges, (2) a supplementary physical model to motivate numerical considerations necessary to obtain a computer implementation of the plasma model, (3) a sketch of the numerical procedure used to calculate the results, and (4) results of a parametric investigation of the phenomenology of ion propagation through electrically conducting fiuids with imbedded, moderately intense magnetic fields (several thousand gauss).

\section{THE PLASMA MODEL}

In practical applicatioris, ICF microexplosions will be initiated by laser or particle beams inside containment vessels filled with a residual fluid. ${ }^{3}$ The bean transmission requirements impose an upper 1 imit on the admissible fluid density $n$. At the present tine this linit is not known precisely, but it is not expected to exceed a value between $10^{15}$ and $10^{16}$ atom/cm ${ }^{3}$. A 
representative volume of an ICF containment vessel (2-m-radius cylinder, $4 \mathrm{~m}$ long) is $5 \times 10^{7} \mathrm{~cm}^{3}$; therefore, the total number of atoms involved may be between $5 \times 10^{22}$ and $5 \times 10^{23}$. If only $0.1 \%$ of the nominal pellet energy release of $150 \mathrm{MJ}$ is absorbed by these atoms, the energy per atom will be greater than $1 \mathrm{eV}$ and therefore sufficient to ionize the medium to a degree that will make it a perfectly conducting plasma.

Under these concitions, the collisional cross sections, $\sigma$, for the fluid-medium particles are approximately ${ }^{4-5} 10^{-15} \mathrm{~cm}^{2}$; consequently, the mean free path $\lambda(\lambda \sim 1 / \pi n \sigma)$ is between 0.30 and $0.03 \mathrm{~cm}$. These estimates show that phenomena with characteristic lengths of several centineters or more can be modeled with a continum description.

However, the fuel pellet debris ions gene:ated in the microexplosion behave differently. Calculations based on the Lindhard stopping theory ${ }^{6}$ show that even at the density of $1.5 \times 10^{16}$ atom/ $\mathrm{cm}^{3}$ (exceeding the allowable $11 \mathrm{mit}$ ), the mean debris-ion range in neon is between 2 and $5 \mathrm{~m}$. Ion ranges of that magnitude are comparable to or greater than characteristic dimensions of ICF containment vessels and much greater than the mean free paths of the background medium. Therefore, the ion motion cannot be approximated within the framework of a fluid model, but must be determined from kinetic theory; in our analysis this is accomplished by calculating trajectories for a statistical sample $(5000$ to 20000$)$ of ions.

To prevent the high-energy fons from striking and eroding the cylinder wall, an axial magnetic field is impressed that deflects their trajectories and guides the ions into the cylinder ends. ${ }^{2-3}$ Preliminary investigations showed that a magretic field intensity of a few thousand gauss will suffice to accomplish this process. 7

Consistent with the estimates made above, the plasma model for the analysis of cavity phenomena consists of three parts: background fluid; discrete, relatively high-energy ions; and an electromagnetic field. The single-component background fluid is postulated to be initially ionized by the flash of $x$ rays emitted from reacting fuel to a degree that makes it a perfectiy conducting plasma. Its behavior is therefore described by modified
Lundquist equations supplemented with an appropriate equation of state and a rate equation that determines changes in the degree of ionization. The expansion of debris ions is described with a representative sample of lon trajectories calculated by integrating the equation of mution for each simulation Ion with the Lorentz force contributing to the acceleration. The model also includes phenomenological terms that describe momentum and energy transfer between the fluid and the discrete ions and a rate equation that determines the average lon charge.

The electromagnetic field is described with Maiwel1's equations in which the displacement current is neglected. Ohm's law is replaced with postulates that the electric field is always and everywhere perpendicular tc the magnetic field, that local charge neutrality prevalls, and that the electrons are massiess and drift without inertia in the direction perpendicular to electric and magnetic fields. Thus, the present model constitutes a generalization and extension of considerations introduced by Dickman, Morse, and Nielson. 8

To be speciffc, we present the differential equations and the constitutive relations that describe the above plasma model. Fr. ronciseness we employ vector representation and write the differential equations in conservation form to facilitate discussion of finite difference approximations. In our notation convention, the operator acts on all factors that follow it.

The conservation of mass, momentum, and energy is ensured by

$$
\begin{aligned}
& \frac{\partial \rho}{\partial t}+\nabla \cdot(\rho u)=0, \\
& \frac{\partial}{\partial t}(\rho u)+(\nabla \cdot u)(\rho u)+\nabla_{p} \\
& +\frac{1}{c} B \times\left(J-J_{1}\right)+\Gamma_{B}=0,
\end{aligned}
$$

and

$$
\begin{aligned}
& \frac{\partial}{\partial t}\left[\rho\left(\frac{1}{2}|u|^{2}+E\right)\right]+(\nabla \cdot u)\left[\rho\left(\frac{1}{2}|u|^{2}+E\right)+p\right] \\
& =E \cdot\left(J-J_{1}\right)+S_{B},
\end{aligned}
$$


and the change in the ionization fraction $\alpha$ is described by

$$
\frac{d \alpha}{d t}=F_{1}(p, T)
$$

In these equations, $D$ is the background fluid mass density, $u$ the velocity vector, $p$ the pressure, $c$ the speed of light in vacuum, 8 the magnetic field vector, $J$ the total current vector, $d_{I}$ the current carried by the debris ions, $E$ the internal energy, $E$ the electric field vector, and $T$ the temperature. The symbol $\nabla$ denotes, as usual, gradient operator, and $|u|$ the magnitude of the vector $u$. The terms $\Gamma_{B}$ and $S_{B}$ represent collisional momentum and energy interchange between the background fluid and the debris ions.

The trajectories of the debris ions are determined $^{2,8}$ by integrating the equations of motion

$$
m_{i} \frac{d v_{i}}{d t}=q_{i}\left(E-\frac{1}{c} B \times v_{i}\right)-\Gamma_{i},
$$

where $m_{i}, v_{i}$, and $q_{i}$ are the mass, velocity vector, and charge of the $i$ th $i o n$, and $\Gamma_{i}$ is the collisional drag force exerted by the background fluid on the ion. In practice, Eq. (5) is integrated for simulation particles that represent a cluster of ions of a given element. The charge of the simulation particle changes according to

$$
\frac{d q_{i}}{d t}=F_{2}\left(\rho, T, m_{i}\right) \text {, }
$$

where $F_{2}$ is the recombination rate for each ion material.

The electromagnetic field is governed by Maxwe 11's equations which, with the previously indicated approximations, reduce to

$$
\frac{\partial B}{\partial t}=-c \nabla \times E
$$

and

$$
\mathrm{J}=\frac{\mathrm{C}}{4 \pi} \nabla \times B
$$

Equations (1)-(8) do not form a closed system and must be supplemented with constitutive relations among different physical quantities. These are of two kinds: those relating the themodynamic variables, $T, P, \varepsilon$, and $p$ (known as equations of state) and those relating the current density $\mathrm{J}$ and the electric field $\mathrm{E}$ (known as Ohm's law).

The themodynamic variables are related by

$$
T=\frac{m(r-1)}{\left(N_{A} \rho+n m\right)^{k}} \rho \varepsilon
$$

and

$$
p=\left(\frac{N_{A} \rho}{m}+n\right) k T,
$$

where $m$ is the atomic mass of background fluid $(\mathrm{g} / \mathrm{mole}), \quad \gamma$ is the ratio of specific heats (assumed constant), $N_{A}$ is Avogadro's number, $k$ the Boltzmann constant, and $n$ the electron density.

Consistent with the previously scated hypothesis about the behavior of the electrons, the expression for the current density $\mathrm{J}$ is

$$
J=J_{I}+\frac{N_{A} e}{m}-Z \text { apu }-\operatorname{nec} \frac{E \times B}{|B|^{2}} \text {, }
$$

where $Z$ is the atomic number of the background rluid, e the magnitude of the electron charge; and $J_{I}$, the debris ion current density, is given by

$$
J_{I}=\sum_{\text {vofume }}^{\text {unit }} q_{i} v_{i} .
$$

From the assumption of charge neutrality, the electron density $n$ is

$$
n=\frac{1}{e} \sum_{\text {votuhe }}^{\text {unit }} q_{i}+\frac{N_{q} p}{m} z \alpha \text {. }
$$

The relation between the current density and the electric field (Ohm's law) may be solved for the electric field $E$; using the postulated orthogonality of the electric and magnetic fields, the resulting expression is 


$$
E=\frac{B}{n e c} \times\left(J_{I}+\frac{N_{A} e}{m} Z \alpha \rho u-J\right) .
$$

Equations (1)-(14) constitute a closed, selfconsistent system describing the plasma model.

In summary, the assumptions implicit in this model are

(1) the background medium is collisiondominated, behaves as an ideal gas with a constant ratio of specific heats, and is ionized to a degree at wich its electrical resistivity may be neglected;

(2) the discrete ions do not directly interact with each other and are not in themodynamic equilibrium with the surrounding fluid in which their ranges are comparable to the characteristic dimensions of interest;

(3) the interaction force between the ions and the surrounding fluid is always tangent to the ion trajectory, that is, scattering is neglected;

(4) there is a sufficient number of sufficiently mobile electrons to ensure local charge neutrality and to short out any electrostatic field;

(5) the electric and magnetic fields are always and everywhere perpendicular; and

(6) high-frequency phenomena are ignored, that is, the displacement current is neglected.

Conditions anticipated in the studies of ICF devices necessary to realize applications of this process are such that these assumptions are mostly satisfied.

\section{ION-FLUID INTERACTION}

\section{A. The Interaction Force $\Gamma_{i}$}

The expression for the force $\Gamma_{1}$ exerted by the fluid on the ion is derived from the concept of the stopping power SP. Denoting by $x$ the distance along the ion trajectory and by KE its (kinetic) energy, we obtain from the definition of the stopping power, $S P=d(K E) / d(\rho X)$, and of the rinetic energy, $K E=(1 / 2) m_{i} v_{i}^{2}$, the expression $\Sigma_{1}=D S P$. For numerical calculations, it is convenient to express SP as the sum of electronte, $S P_{e}$ and nucleonic, $S P_{n}$, contributions, that 15 ,

$$
\Gamma_{1}=\rho\left(S P_{e}+S P_{n}\right)
$$

The stopping powers of different media have been extensively investigated both theoretically and experimentally. Various physical mechanisms may dominate or contribute, depending on the range of parameters in which the calculations are performed. Examples of mechanisms relevant to ICF application studies are excitation and ionization of the background fluid (Bethe-Bloch processes), Coulamb interactions between debris and background ions, and coulomb interactions between debris ions and free electrons. The proper expressions to be used in Eq. (15) depend on the conditions prevailing in the cavity and on the desired degree of approximation.

Consistent with the sign convention in Eqs. (2) and (5), the reaction farce on the background medium $\Gamma_{B}$ is the sum of $\Gamma_{j}$ over the number of ions present in each unit volume. In calculations of the forces and of the ion energy loss $S_{B}$, the relative velccity between the ions and the background medium is used.

Figure 1 shows a typical dependence on the ion energy of the force $\Gamma_{i}$ retarding the motion of a tungsten ion traveling through un-ionized argon at a density of $10^{-8} \mathrm{~g} / \mathrm{cm}^{3}$; the shapes of such curves for different ions are very similar.

\section{B. Qualitative Aspects of Ion-Fluid Interaction}

Before proceeding with the calculations of solutions of Eqs. (1)-(14), it is instructive to examine with an elementary model the propagation

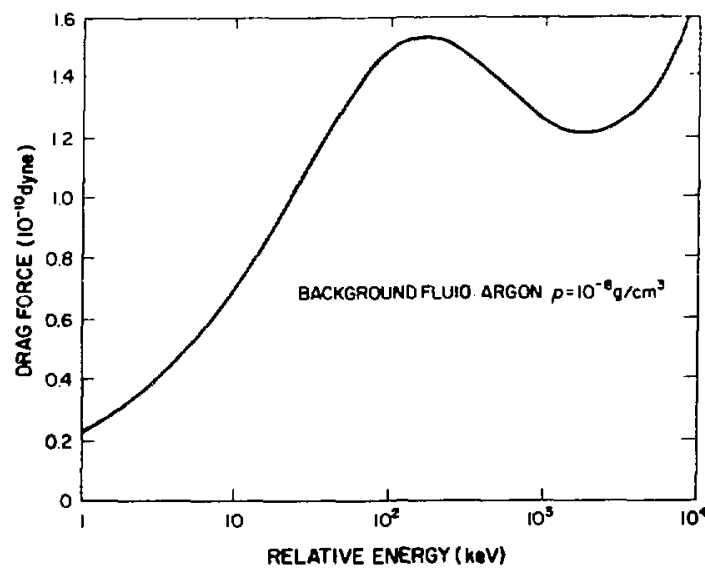

Fig. 1. Drag force on a tungsten ion. 
of tons through fluids. The insight gained from such investigation is helpful in the implementation of finite difference calculations and in the interpretation of results.

The elementary model studied in this paper is described by the two Newton's equations of motion for the masses of ions and fluid per unit volume:

$$
\frac{d v_{i}}{d t}=-\operatorname{sgn}\left(v_{i}-u\right) \frac{\Gamma_{0} \rho}{\sqrt{\pi_{i}}}\left|v_{i}-u\right|^{\beta}
$$

and

$$
\frac{d u}{d t}=\sum_{i} \operatorname{sgn}\left(v_{i}-u\right) n_{i} \sqrt{m_{i}} \Gamma_{0}\left|v_{i}-u\right|^{B},
$$

where the constants $\Gamma_{0}$ and $B$ are determined to locally approximate the behavior shown in Fig. 1, and the subscript $i$ distinguishes between individual ions. The presence of the function $\operatorname{sgn}(\cdot)$, which denotes the sign of the difference $\left(v_{i}-u\right)$, indicates that the ion is accelerated by the fluid whenever its velocity $v_{i}$ is less than the fluid velocity $u$.

Equations (16) and (17) were integrated numerically with the following initial conditions: at $t=t_{i}, v_{i}=v_{i 0}$, where $\left\{t_{i}\right\}$ is an arbitrarily specified sequence of increasing numbers, $t_{i}$ being the time when the $i^{\text {th }}$ ion begins to move; and $\left\{v_{i 0}\right\}$ is a set of randomly generated numbers in the range $0.5 \times 10^{7} \leq v_{i 0} \leq 1.5 \times 10^{7} \mathrm{~cm} / \mathrm{s}$. We set $t_{1}=0$ and also prescribe $u=0$ at $t=0$.

For $\beta=1$, the system is linear and may be solved explicitly. The result shows that the solution depends only on the mass ratio of the ions to the entrained fluid in the interaction volume, $n=m_{\mathbf{i}} n_{\mathbf{i}} / \rho$. We present numerically calculated results for low $(n=0.1)$, intermediate $(n=1.0)$, and high $(n=100)$ values of the mass ratio; in all cases a sample of ten ions was used with initial times uniformly distributed over the characteristic interaction time whose value was estimated from the solution of the linear equations $(B=1)$.

Figure 2 is a plot of ion velocities (solid curves) and ion trajectories (dashed curves) for $\eta=0.10$ and $\beta=1.10$. It shows that the ion interaction with the background medium rapidly reduces ion velocities to constant values, and

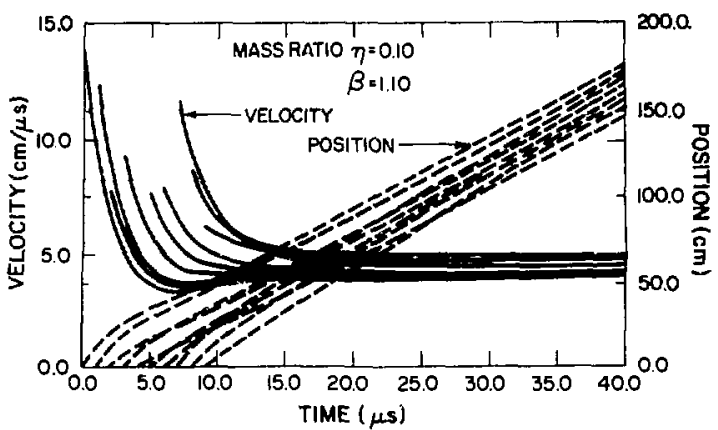

Fig. 2. Ion velocities and trajectories for low mass ratio.

therefore the ion trajectories do not spread even though the initial ion velocities were randomly distributed. The same results are shown in a more concise form in Fig. 3 ; here the solid curve represents the average (over i) ion velocity and the dashed curve the background fluid velocity $u$. The discontinuities occur when new ions with random velocities are introduced.

Figure 4 shows the behavior of the average ion velocity and of the background fluid velocity for $\eta=1.0$ and $B=1.0$; as may be expected, the two velocities asymptotically neet approximately halfway between the zero and maximum initial ion velocity of $1.5 \times 10^{7} \mathrm{~cm} / \mathrm{s}$.

Figure 5 shows the individual ion velocities and trajectories for $n=100$ and $\beta=1.30$. Because of the large ion mass, the background fluid does not affect the ion trajectorits; the ion velocities remain approximately corstant, and their

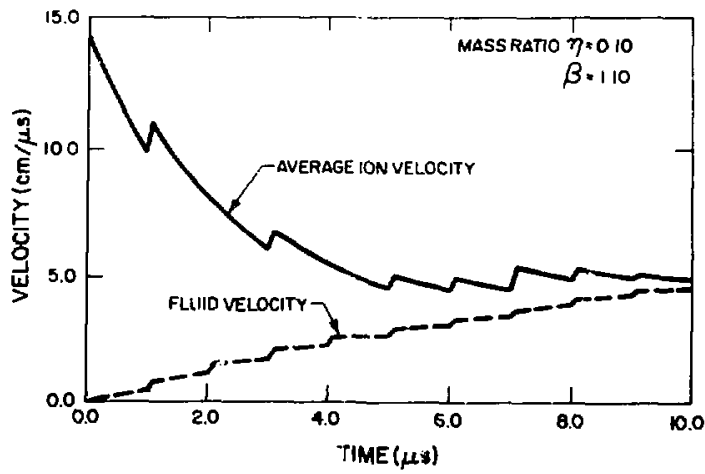

Fig. 3. Fluid and average fon velocities for low mass ratio. 


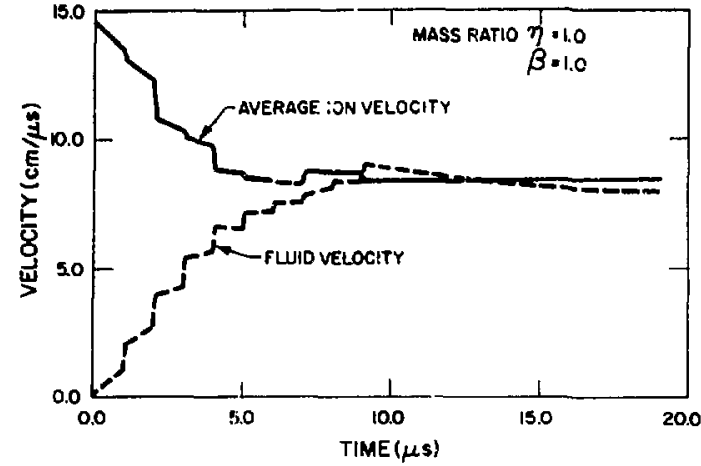

Fig. 4. Fluid and average ion velocities for intermediate mass ratio.

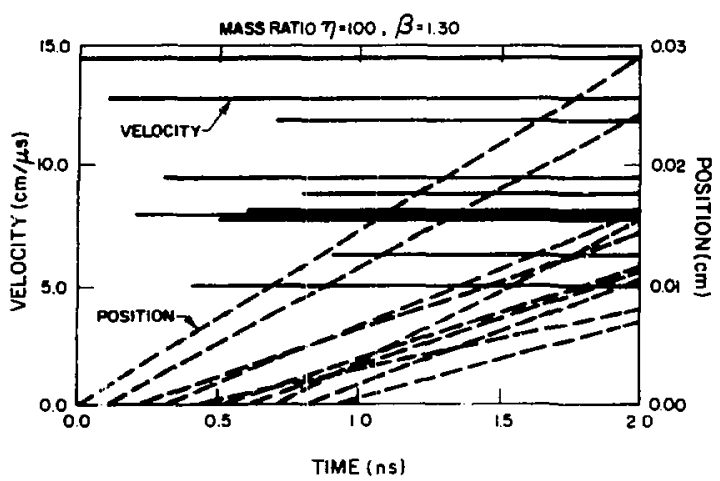

Fig. 5. Ion velocities and trajectories for high mass ratio.

trajectories show the corresponding time-of-flight spreading on the time scale of the plot.

The results 111 ustrated in Figs. 2-5 will be used in the computation and interpretation of the solution of Eqs. (1)-(14).

\section{IY. THE INTEGRATION PROCEOURE}

In studies of ICF applications, particulariy in analyses of explosion containment vessel requirements, we are interested in the time evoiution of plasm conditions. It is described by the solution of the inttial value problem for Eqs. (1)-(14) inside a cylindrical domain bounded by electrically conducting walls. Therefore, the bosindary conditions are the vanishing of the tangential component of the electric field and of the nomal component of mass velocity and the continuity of the tangential component of the magnetic field. Because the events following pellet microexplosion occur in times that are short in comparison to the characteristic time for the magnetic diffusivity in the wall material, it is appropriate to require al so that the magnetic field at the boundary does not change.

Dur plasma model does not contain viscosity and heat conductivity effects; therefore, the above-stated boundary conditions are the only requirements imposed by the physical considerations. However, to make the inttial-boundary value problem numerically well-posed, it is necessary to specify boundary conditions for all primary variables, that is, those whose time rate of change is given by differential equations. To obtain these additional conditions we anticipate that the computed phenomena will consist predominantly of wave reflections and postulate that the reflections wil be nearly normal. Consequently, the vanishing of the nomal derivative at the boundary should be approximately valid locally for pressure, density, and the tangential component of the velocity. We also postulate that the ions adhere to the wall upon striking it; this is achieved by removing the ion from the computational scheme whenever its trajectory intersects the boundary. A record of the time, position, and fon energy at the time of inpact is retained for use in anaiyses of wall heating and erosion. ${ }^{9}$

Solutions describing the evolution of plasma conditions inside reactor cavities are obtained by numerical integration of the governing equations. Toward this end, differential Eqs. (1)-(4) and (7) are replaced with finfte difference approximations using the Lax-Friedrichs scheme introduced by Lax ${ }^{10}$ and employed effectively by Bohachevsky and collaborators $11-13$ to valculate miltidimensional flows with shock waves and radiation effects. Equation (5) is integrated straightforwardly in time, and the auxiliary variables are evaluated at each time step and snoothed with a four-point averaging process to ensure a stable and accurate comutation.

Exploratory calculations and supporting analysis of ion-fluid interaction presented in Sec. III showed that the relaxation time for the 
transfer of momentum and energy from the ions to the flutd is much shorter than the characteristic fluid-dynamic time because, in the early stages of the expansion, the mass ratio of the ions to the entrained fluid in the interaction volume is much larger than unity. Therefore, the complete set of the governing difference equations is stiff; the degree of stiffness may be appreciated from the fact that while the characteristir interaction time may be as small as $10^{-12} \mathrm{~s}$, the typical characteristic fluid-dynamic time is approximately $10^{-6} \mathrm{~s}$. The associated computational difficulties were resolved using the results of Sec. III, which indicate that in the computational cells in which the mass ratio exceeds 10 ( $n \geq 10$ ), the fluid velocity and internal energy should equal the average values of these variables for the ions present in the cell.

The characteristic time for the ionization and recombination processes governed by Eq. (4) also may be very short in comparison to the integration time step determined by the fluid dynamical phenomena. In that case it is convenient and appropriate to use, in place of Eqs. (4) and (9), relations based on the Saha equation. These can be of two types: numerically calculated and tabulated compatible values of ionization fraction $\alpha_{\text {, internal }}$ energy $r$, and temperature $T$, or analytic approximations. An example of the latter applicable to the study of ICF plasma phenomena is the coronal model of shearer and Barnes: 14

$$
a=\frac{26}{Z}\left[\frac{k T(k e V)}{1+(26 / 2)^{2} k T(k e V)}\right]^{1 / 2},
$$

which satisfactorily approximates experimental results in paraneter ranges of our interest.

The integration is incitalized by (1) determination of the desired magnetic field distribution (either by placing coils at appropriate locations or by solving the bounda $/$ value problems for the magnetic vector potential), (2) placenent of simulation particles into desired locations with desired velocities (energies), and (3) prescription of initial distributions for the density, velocity, and temperature of the background fluid.
After initialization, the variables are advanced in discrete time steps in the following order:

Eq. (5):

(1) the simulation particles are moved using

(2) the values of density, velocity, and internal energy of the fluid are advanced using Eqs. (1)-(3);

(3) the auxiliary thermodynamic quantities (including ionization fraction $\alpha$ ) are determined;

(4) the current is calculated with Eq. (8);

(5) the electric field is calculated with Eq. (14); and

(6) the magnetic field is advanced with Eq. (7) completing the cycle.

The computations are continued until all simulation particles disappear into containment walls or a quasi-steady state has been reached.

\section{Y. NLMERICAL RESULTS}

Equations (1)-(14) were integrated numerically inside a cylindrical domain with conical end boundaries. The cylinder radius was $250 \mathrm{~cm}$ and the cylinder length was $1250 \mathrm{~cm}$ at the axis and $750 \mathrm{~cm}$ at the wall, that is, the half-angles of the end-cones were $45^{\circ}$. Because of the axial and central plane symetries, only one quarter of the domain, show in Fig. 6, was covered with the computational mesh. The mesh size was $\Delta r=\Delta z=5 \mathrm{~cm}$.

$$
\begin{gathered}
\text { I IME }=1.2 E-05 \\
P_{0}=3.44 \times 10^{-}
\end{gathered}
$$

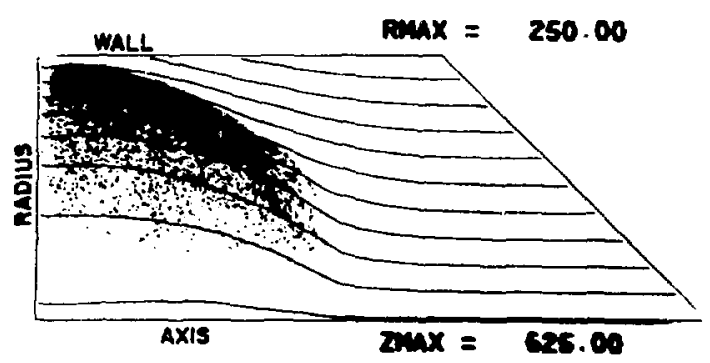

Fig. 6. Ion cloud in fluid density of $n=10^{14}$ 
The initially uniform number density of the fluid medium was varied from $10^{14}$ to $10^{17} \mathrm{~cm}^{-3}$ at the temperature of $1000 \mathrm{~K}$. This corresponds to the pressure range from 1.72 to $1.72 \times 10^{3} \mathrm{dyn} / \mathrm{cm}^{2}$ and the density range from $3.44 \times 10^{-9}$ to $3.44 \times 10^{-6}$ $g / \mathrm{cm}^{3}$ for lead vapor. The initial ionization fraction was assumed to be 0.003 and the ratio of specific heats was 1.05. An initially uniform axial magnetic field of 5000 gauss was imbedded in this medium.

The ions were initially randomly distributed inside a few (9-25) cells near the origin $(r=z=0)$. Their initial velocity directions were randomly distributed on the unit hemisphere and the initial velocity magnitudes ranged from $0.1 v_{\max }$ to $v_{\max }$ with the distribution given by $n(v) d v{ }^{\max }=3 n_{\text {tot }}\left(v^{2} / v_{\max }^{3}\right) d v$. The value of $v_{\text {max }}$ was determined from the total initial ion energy content, which was $2.25 \times 10^{14} \mathrm{erg}$ in our computations.

The initial value of the background fluid temperature in those cells in which the ions were initially located was $2 \times 10^{7} \mathrm{~K}$, and the density was ten times higher than the ambient value. The computations, therefore, simulated the propagation of the ions and of the blast waves.

The results show markedly different behaviors in the low $\left(10^{14}-10^{15} \mathrm{~cm}^{-3}\right)$ and in the high $\left.110^{16}-10^{17} \mathrm{~cm}^{-3}\right)$ ambient density cases.

Figures 6-9 illustrate the characteristics of the ion expansion through tenuous $\left(10^{14}-10^{15} \mathrm{~cm}^{-3}\right)$ media. The relatively extensive dispersion of ions (Figs. 6 and 8 ) and the smooth variation of the magnetic field intensity (Figs. 7 and 9 ) are significant. The lines in Figs. 6 and 8 are contours of constant magnetic flux.

Figures 10-13 illustrate the characteristics of the ion expansion through relatively dense $\left(10^{16}-10^{17} \mathrm{~cm}^{-3}\right)$ media. The concentration of ions in thin sheets (Figs. 10 and 12) and the associated discontinuities in the slope of the magnetic field intensity (Figs. 11 and 13) are significant. Such behavior is consistent with the results presented and discussed in Sec. III, which indicate that the dominant effect in ion-fluid interaction is the dependence of the interaction force on the fluid density and on the relative velocity between the ion and the fluid. This dependence equalizes

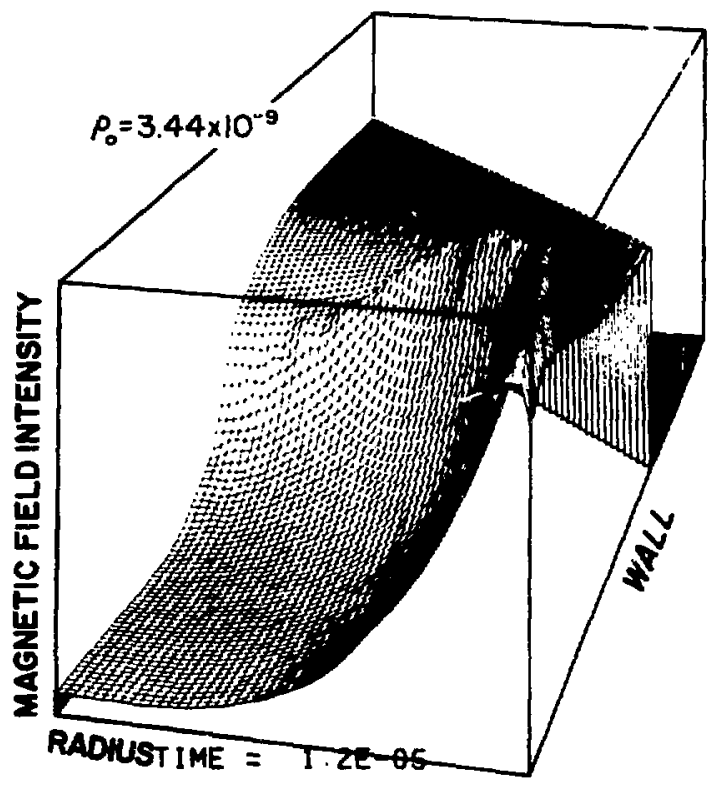
Fig. 7. Magnetic field jntensity in fluid density
of $n=1074 \mathrm{~cm}^{-3}$.

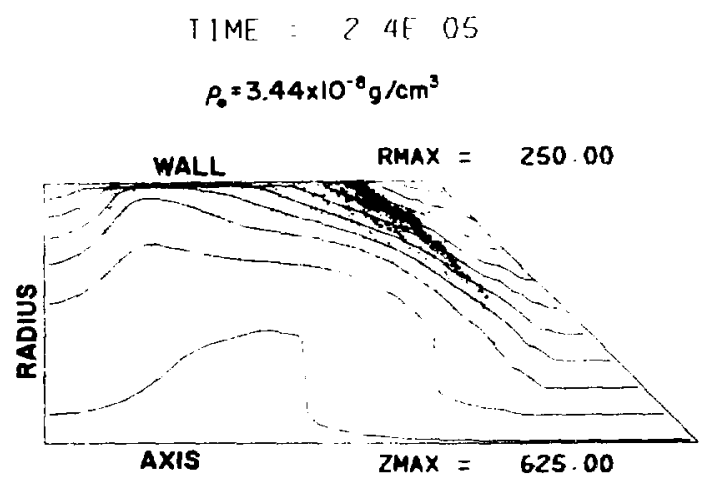

Fig. 8. Ion cloud in fluid density of $n=10^{15}$ 


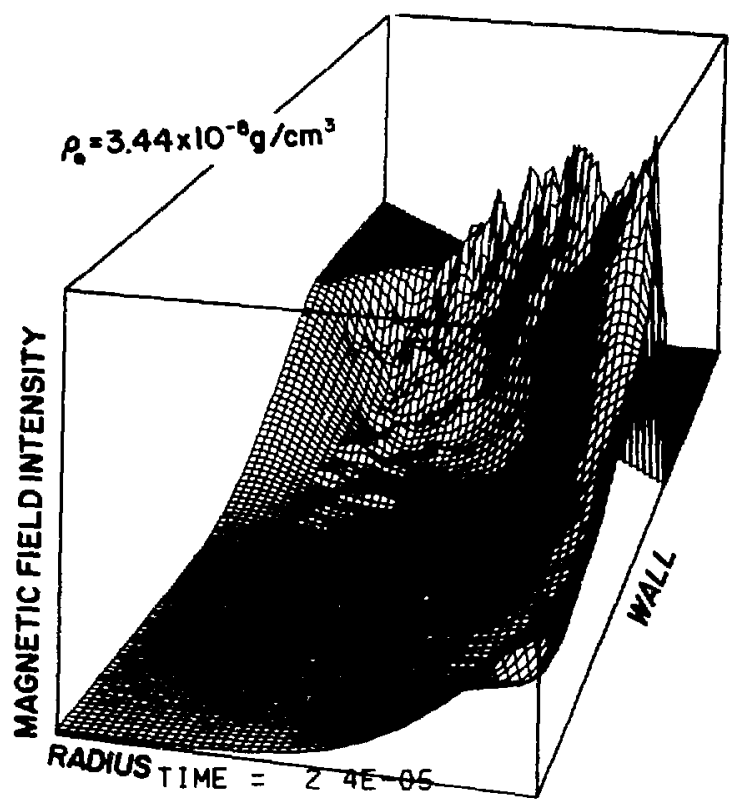
Fig. 9. Magnetic field intensity in fluid density
of $10^{15} \mathrm{~cm}^{-3}$.

$$
\begin{aligned}
& \text { TIME }=23 E-0 S \\
& P_{0}=3.44 \times 10^{-7} \mathbf{g} / \mathrm{cm}^{3}
\end{aligned}
$$

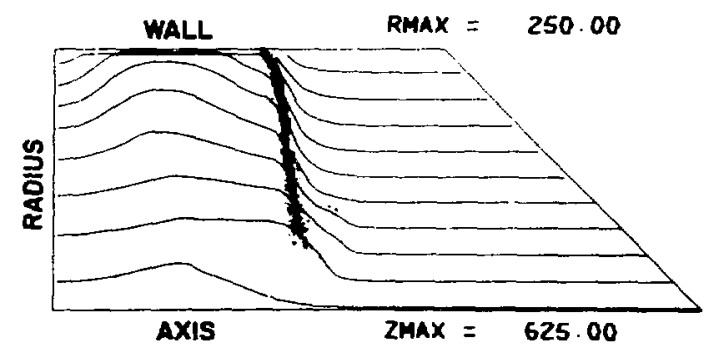

Fig. 10. lon sheet in fluid density of $n=1016$

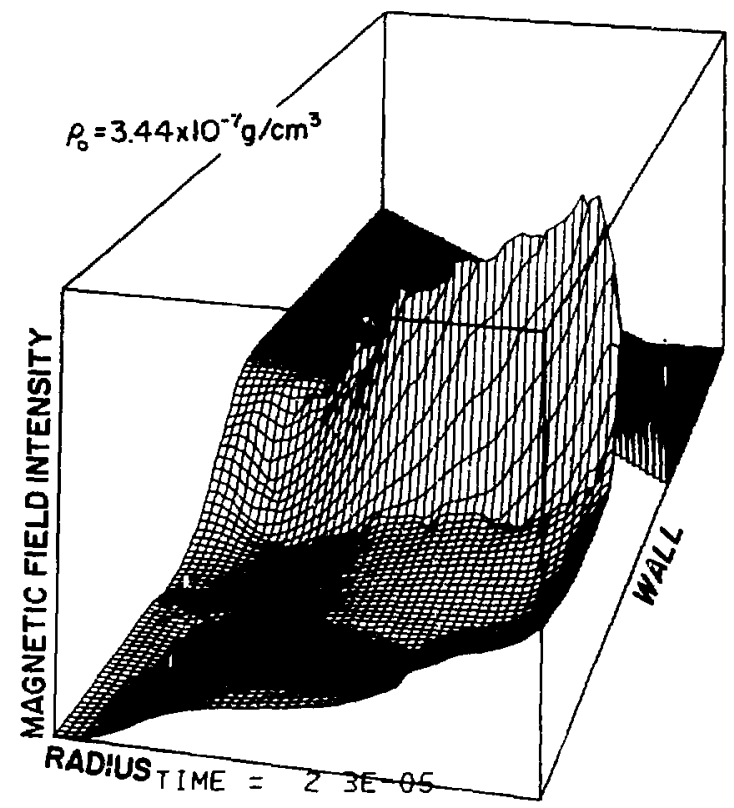
Fig. 11. Magnetic fjeld jntensity in fluid density

$$
\begin{gathered}
\text { TIME }=24 E-05 \\
\rho_{0}=3.44 \times 10^{-6} \mathrm{~g} / \mathrm{cm}^{3}
\end{gathered}
$$

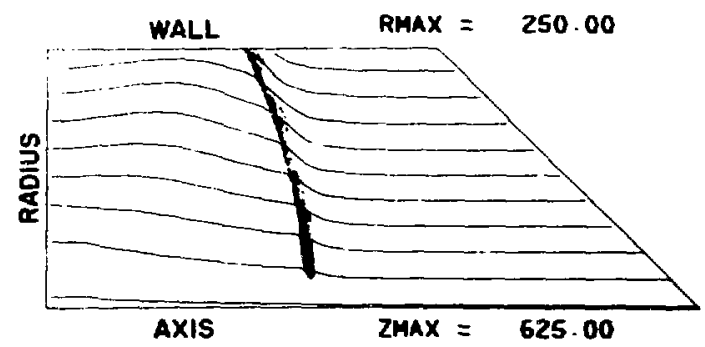

F1g. 12. Ior sheet in fluid density of $10^{17} \mathrm{~cm}-3$. 


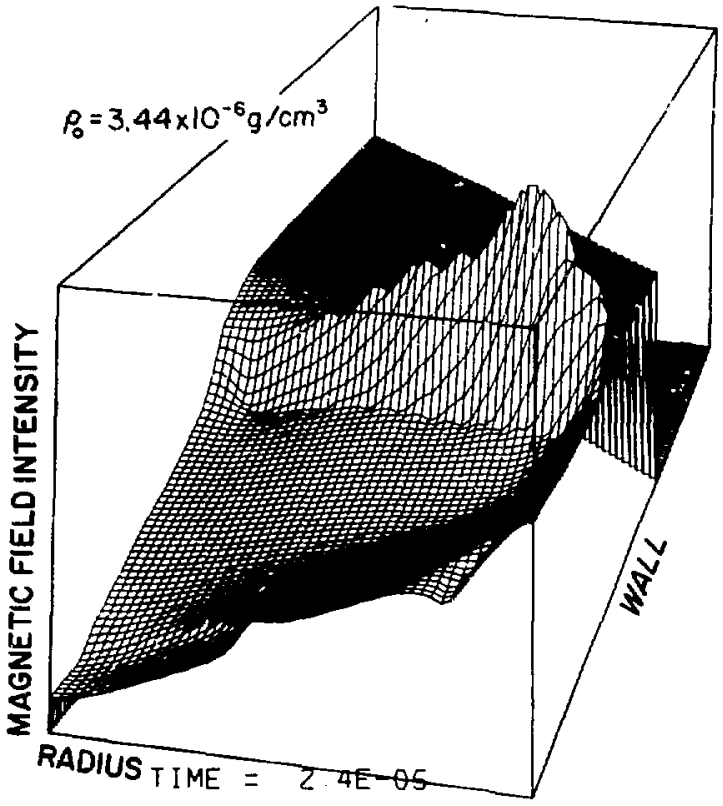

Fig. 13. Magnetic field intensity in fluid density of $n=10^{17} \mathrm{~cm}^{-3}$.

all velocities at a rate that increases with the increasing fluid density.

Figures 14 and 15 illustrate the pressure and the axial fluid velocity distributions for the ambient density of $3.44 \times 10^{-8} \mathrm{~g} / \mathrm{cm}^{3}$. The maximum values of these variables at the times of the plots were $7 \times 10^{4} \mathrm{dyn} / \mathrm{cm}^{2}$ and $1.6 \times 10^{7} \mathrm{~cm} / \mathrm{s}$. These results show that the ions impart such a high velocity to the background fluid that, in spite of iarge energy deposition, the temperature and density in the region interior to the expanding ions cecrease to very low values. Thus, the individual ions act analogously to jet ejectors and the effect is quite strong: it reduces the density inside the expanding ion shell to a value less than one one-chousandth of the initial ambient density.

\section{CONCLUDING OBSERVATIONS}

In general, the solutions indicate that the background fluid has only a modest influence on the speed of the propagating ions. In al! computations the maximum initial ion velocity was

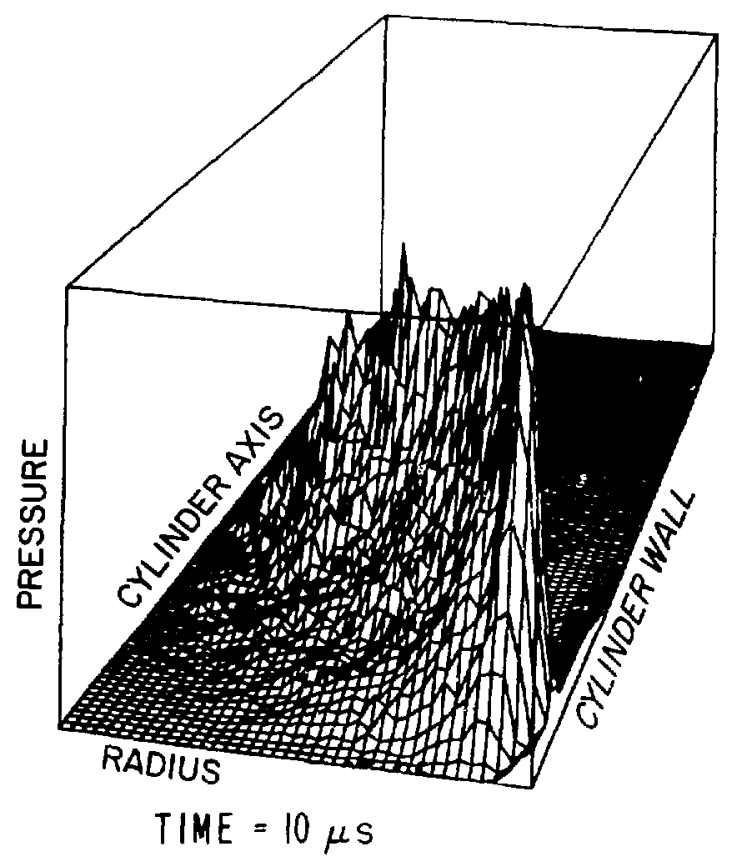

Fig. 14. Fluid pressure at $t=10: 5$.

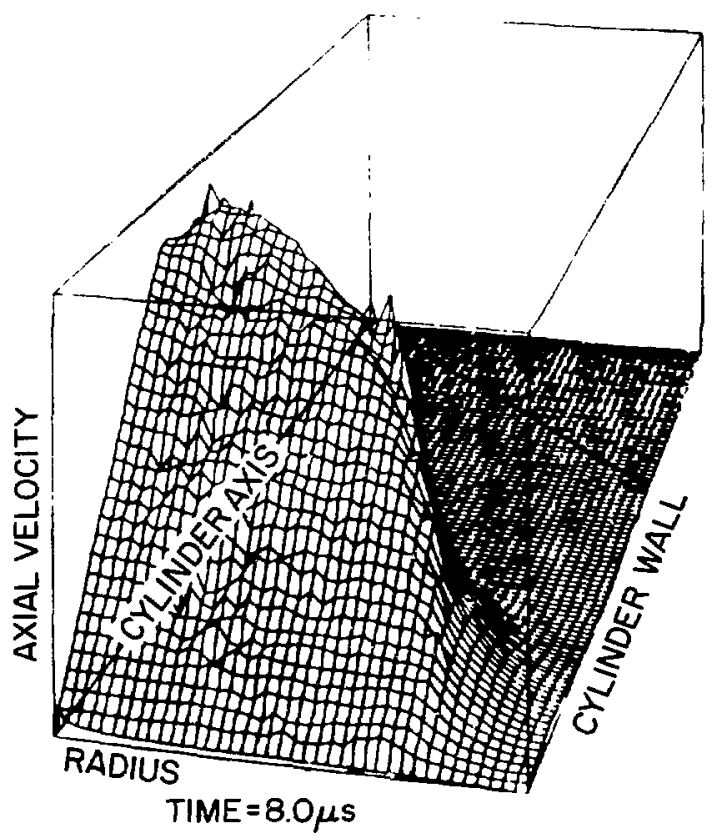

Fig. 15. Axial component of the fluid velocity at $t=8 \mu \mathrm{s}$. 
$v_{\max }=2 \times 10^{7} \mathrm{~cm} / \mathrm{s}$; therefore, in a vacuum the ions would reach the cylinder wall in $12.5 \mu \mathrm{s}$. This value agrees closely with the value estimated from Fig. 6 for the low-density case of $10^{14} \mathrm{~cm}^{-3}$ $\left(3.44 \times 10^{-9} \mathrm{~g} / \mathrm{cm}^{3}\right)$ and suggests that our mode) does not distinguish such a low density from the vacuum. At the background density of $10^{16} \mathrm{~cm}^{-3}$ $\left(3.44 \times 10^{-7} \mathrm{~g} / \mathrm{cm}^{3}\right)$ the fons require $17.5 \mu \mathrm{s}$ to approach the wall, which indicates an approximately $30 \%$ reduction in speed for a factor of one hundred increase in the density.

Similarly, Figs. 10 and 12 show that the average axial ion velocity, $v_{z a v}$, is approximately $10^{7} \mathrm{~cm} / \mathrm{s}$, which is an approximately $30 \%$ reduction from the initial ayerage ion velocity of $v_{\text {iav }}=3.5 \times 10^{7} \mathrm{~cm} / \mathrm{s}$.

However, the results indicate that the background fluid significantly affects the configuration of the expanding ion cluster. Figures 6,8 , 10, and 12 show that an increasing fluid density changes the relatively diffuse ion clouds into well-defined thin sheets oriented perpendicularly to the magnetic field direction and propagating along the magnetic field lines.

The results also appear to indicate that the background fluid significantiy affects the magnetic field compression. Apparently, the presence of the infinitely conducting background medium attaches "inertia" to the magnetic lines of force and thus inhibits their compression. Thus, the magnetic field compression decreases with increasing background fluid density as shown in Fig. 16, where the time history of the ratio of highest, $|B|_{\max }$, to the initial, $|B|_{O}$, magnetic field intensity along the radius at $z=25 \mathrm{~cm}$ is plotted. The decrease, however, is reversed at higher densities where the ions tend to assemble into thin sheets as indicated by the curve labeled $\rho_{0}=3.44 \times 10^{-7}$ $\mathrm{g} / \mathrm{cm}^{3}$. We conjecture that conducting fluids at low densities have sufficient inertia to inhibit magnetic field compression, but insufficient stopping power to affect the ion motion significantly, whereas at higher densities the ions aggregate into sheets that compress the magnetic field against the wall like perfectly conducting pistons. However, our computations are neither sufficiently accurate nor sufficiently

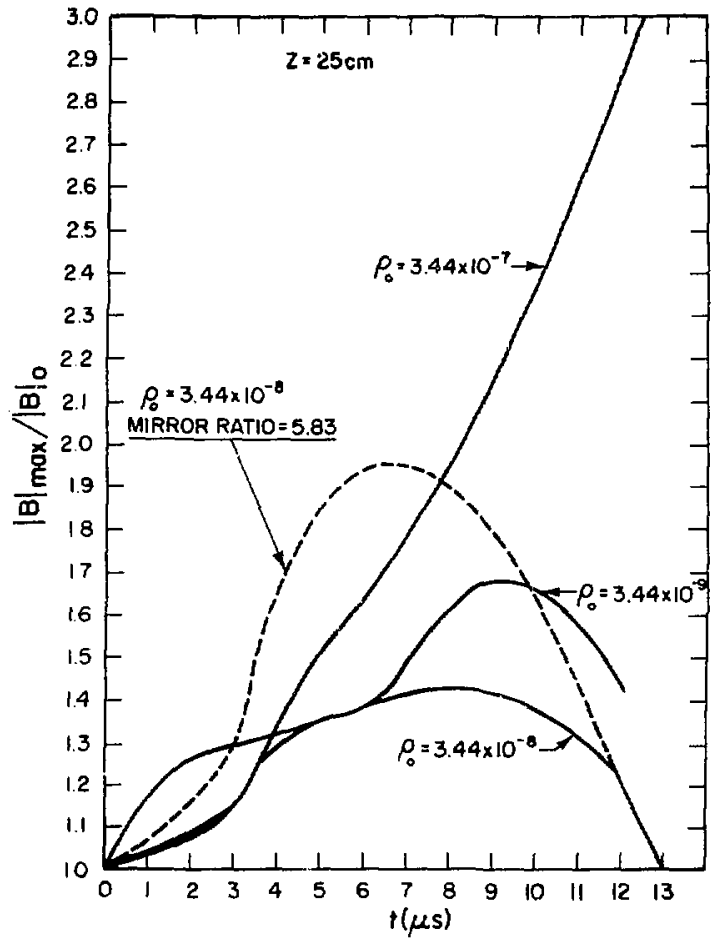

Fig. 16. History of the magnetic field compression.

extensive to establish such cehavior unequivocally. Additional analytical and numerical investigations are needed to gain full understanding of this phenomenon.

The dashed curve in Fig. 16 illustrates how the mirror configuration of the initial magnetic field accelerates and enhances the magnetic flux compression by delaying the ion escape; the initial value of the mirror ratio in this computation was 5.83 .

\section{REFERENCES}

1. I. O. Bohachevsky, J. C. Goldstein, and D. 0 . Dickman, "A Plasma Model for ICF Reactor Studies," Proc. of the International Conf, on Plasma Physics, Magoya, Japan, April 7-11, 1980 (Fusion Research Association of Japan), Vol. 1, p. 384 .

2. I. 0. Bohachersky, J. C. Goldstein, and D. 0 . Dickman, "Plasma Behavior in Magnetically Protected Inertial Confinement Fusion Reactor Cavities," Huc1. Tech./Fusion 1, 390-401 
3. I. 0, Bohachersky, "Models and Analyses for ICF Reactor Studies," Los Alamos National Laboratory report LA-8557 (May 1981).

4. J. L. Delcraix, Introduction to the Theory of Ionized Gases Interscience PubTishers, New York, 1960), pp. 19-22.

5. S. Chapman and T. G. Cowling, The Mathematical Theory of Nonuniform Gases TCambridge University Press, $19521, \mathrm{pp}, 220,252$.

6. R. H. Conn, S. I. Abdel-Khalik, G. A. Moses, F. Beranek, E. T. Cheng, G. H. Cooper, R. B. Droll, T. Henderson, J. Howard, T. O. Hunter, E. M. Larsen, G. L. Kuicinski, E. G, Lovell, G. R. Magelssen, C. H. Maynard, K. R. Okula, H. Ortman, H. H. H. Ragheb, K. B. Rensel, D. Solomon, R. L. Spencer, I. N. Sviatoslavsky, W. F. Yogelsang, R. D. Watson, and W. G. Wolfer, "SOLASE, A Conceptual Laser Fusion Reactor Design," University of Wisconsin Fusion Research Program repart UWFDM-220 (December 1977).

7. J. C. Goldstein, I. O. Bohachersky, and D. 0. Dickman, "Plasma Evolution in a Gas-Buffered ICF Reactor Vessel," APS Sulletin, 24 (8), 1071, paper $7 \times 4$ (October i979).

8. D. O. Dickman, R. L. Morse, and C. W. Nielson, "Numerical Simulation of Axisymmetric, Collisionless, Finite- $\beta$ Plasma," Phys, Fluids 12, $1708-1716(1978)$.
9. I. 0. Bohachevsky and J. F. Hafer, "Sputtering Erosion in Inertial Confinement Fusion Reactors," Nucl. Tech. 41 (3), 299-311 (December 1978).

10. P. D. Lax, "Weak Solutions of Nonl inear Hyperbolic Equations and Their Numerical Computation," Commun. Pure Appl. Math. VII, 759-193 (1954).

11. I. 0. Bohachevsky and E. L. Rubin, "A Direct Hethod for Computation of Nonequilibrium Flows with Detached Shock Waves, "AIAA J. 4, 600-607 (1966).

12. I. 0. Bohachevsky and R. E. Mates, "A Direct Method for Calculation of the Flow About an Axisymetric Blunt Body at an Angle of Attack," AIAA J. 4, 776-782 (1966).

13. I. 0. Bohachevsky and R. N. Kostoff, "Supersonic Flow Over Convex and Concave Shapes with Radiation and Ablation Effects," AIAA J. 8, 1024-1031 (1972).

14. J. W. Shearer and W. S. Barnes, "Numerical Calculations of Plasma Heating by Means of Subnanosecond Laser Pulses," in Laser Interaction and Related Plasma Phenomena, $H . J$. Schwarz and H. Hora, Eds. (PTenum Press, New York, 1971) Vol. 3, pp. 307-337. 